\title{
MORGANA, LA HERMANASTRA MALVADA / MALTRATADA DE ARTURO
}

\author{
MORGAN, THE EVIL SISTER / ABUSED BY ARTURO
}

Helena Seoane

Universidad de Oviedo

\section{Resumen:}

La escritora inglesa Rosalind Miles en su libro Ginebra, el primer libro de la trilogía de la saga artúrica junto con Lanzarote, el caballero del Lago Sagrado y Galahad, el hijo del Santo Grial, plantea analizar el papel de Morgana y de Ginebra y la dicotomía que se presenta entre las tradiciones paganas defendidas por ambas frente a la expansión del cristianismo representado por Arturo y Merlín. Una gran parte de las novelas de la materia artúrica ponen de relieve la visión andrógina de la sociedad. Sin embargo, la autora, mediante la trilogía, expone una versión totalmente diferente del papel de los personajes femeninos. Las mujeres que nos muestran las obras, concretamente la que nos ocupa, son fuertes, independientes y no sometidas a las designios del hombre y a través de ella Rosalind Miles nos proporcionará una nueva perspectiva femenina del mito artúrico.

\section{Palabras clave:}

Ginebra, Morgana, matriarcado, diosa, Rosalind Miles.

\section{Abstract:}

The English writer Rosalind Miles in Guenevere, Queen of the Summer Country, the first book of The Guenevere trilogy with The Knight of the Sacred Lake and The Child of the Holy Grail, analyzes the role of Morgan and Guenevere and the dichotomy between pagan traditions espoused by the two women against the spread of Christianity represented by Arthur and Merlin. Many of the novels around Camelot and King Arthur emphasize the androgynous vision of society. However in every book of this trilogy, Miles presents a completely different version of the role of the female characters. They are strong and independent women, not subjected to the designs of men. Miles allows us to access to a new female perspective of the Arthurian myth.

\section{KeYWORDS:}

Guenevere, Morgan, Rosalind Miles, matriarchy, goddness. 


\section{RosAlind Miles y GiNEBRA}

Rosalind Miles es una reconocida autora inglesa que ha publicado veintitrés libros de ficción y no ficción. Ha realizado sus estudios universitarios en la Universidad de Oxford, donde ha obtenido cinco licenciaturas. Inicialmente se interesó por la literatura shakesperiana, y posteriormente por los estudios de género. Sus novelas históricas sobre las reinas británicas han sido internacionalmente aclamadas, destacando la historia de la reina Elizabeth I. Sus siguientes trabajos, las novelas de Camelot basadas en las reinas célticas de la saga artúrica, Ginebra e Isolda, han sido también best-sellers en Gran Bretaña, EEUU y en numerosos países. Sus publicaciones de no ficción incluyen críticas literarias y comentarios sociales sobre el poder y el liderazgo, las mujeres y el trabajo, la masculinidad y el sexo.

La trilogía de Camelot, cuyo primer libro es Ginebra, es una crítica a la política de expansión de la Iglesia católica que ha llevado a la destrucción de las tradiciones célticas, a la sumisión de la mujer y a un atraso económico, social y científico. "Allí donde nosotras vemos fe y amor, ellos ven a un único Dios. En sus escrituras, Él mismo se describe como un Dios celoso y se jacta de que no admitirá a otros dioses ante él. En su Libro Sagrado, enseña a sus seguidores los méritos de la cólera y la destrucción" (Miles, 2013: 250).

En este primer volumen se describe una sociedad matriarcal en la cual Ginebra hereda el reino de Camelot y la Tabla Redonda a través de su madre. Como cualquier reina del País del Verano necesita tomar un caballero de entre los de la Tabla Redonda y convertirlo en rey. Gracias a las conquistas realizadas por Arturo, este se convierte en el paladín de Ginebra y, a su vez, en rey. Morgana desempeñará un papel importante en el desarrollo de la relación entre los dos. Intentará romper esta unión con el objetivo de vengarse de su hermanastro Arturo, del padre de ambos, Uther, y de Merlín, ya que estos últimos la habían encerrado en un convento para toda la vida. Morgana también representará el declive de Arturo y, en consecuencia, de la relación amorosa con Ginebra, del Reino del Verano.

En esta obra no es el amor infiel entre Lanzarote y Ginebra lo que desencadena el final de Camelot, sino la relación incestuosa entre Arturo y su hermanastra Morgana.

El personaje de Lanzarote aparece descrito como el mejor caballero del mundo, convirtiéndose, más adelante, en el caballero de Ginebra. Con él, conocerá el amor verdadero, aunque ella, como reina, elegirá su deber por encima de su amor. Lleva a Arturo a Ávalon para que se recupere de sus "heridas" y así consiguen que sus reinos sigan unidos y libres.

\section{Morgana la Fay}


El libro de Ginebra presenta una defensa del feminismo mediante una visión diferente de los personajes literarios de Ginebra y Morgana, y mediante la crítica de la sociedad patriarcal que realiza la autora a través de ellas, oponiendo la tradición pagana frente a la cristiana.

Se ha resaltado sobre todo el personaje de Morgana porque representa el símbolo de la libertad de la mujer respecto a su cuerpo, a su vida y a sus pensamientos. En primer lugar, se hablará de su personaje dentro del libro, dando paso a la evolución de su imagen desde su primera aparición en las obras medievales, concluyendo con una comparación opuesta a su hermanastro Arturo.

\subsection{Presentación del personaje en Ginebra}

En las primeras páginas del libro, Morgana aparece descrita, por un lado, como una mujer hermosa, con un cuerpo bonito y sensual y se señala que ningún hombre podría escaparse a sus encantos; pero, por otro, ya se nos muestra con tildes de pequeña bruja. Es hija de la reina de Cornualles y estuvo encerrada durante veinte años en un convento por orden de su padre, Uther Pendragón y de Merlín. En esta época los padres tenían dos opciones en cuanto al destino de sus hijas: o la entregaban en matrimonio o era ingresada en un convento donde podría vivir una vida irrefutable -en algunos casos, los padres no deseaban pagar la dote por ella. En este orden de cosas a Morgana se la relega al claustro.

Ginebra se opone a que Morgana viva toda su vida bajo la influencia del cristianismo, por lo que Arturo decide ir a buscar a su hermanastra para que viva con ellos en Camelot.

Morgana, al principio en el Reino del Verano, no hablaba con nadie y vagaba por el castillo como un fantasma. Su madre, Igraine, decía que poseía dotes adivinatorias desde la infancia: "La Diosa había bendecido a Morgana en su cuna. Ya de niña, se comunicaba con los Puros, y la gente la llamaba el hada Morgana. Algunos creían que sus poderes la convertirían en señora de Ávalon cuando llegara el momento" (Miles, 1999: 214).

\subsection{La evolución de La imagen de Morgana}

Su figura ha sufrido innumerables cambios desde su aparición en los textos del siglo XII y, más concretamente, en los del ciclo artúrico: desde una sabia, sanadora y cuidadora ya sea de las mujeres, ya sea del rey Arturo, hasta la bruja más pérfida que intenta acabar con el reino de Camelot y de su rey.

Se la menciona por primera vez en la obra de Geoffrey de Monmouth, Historia de los reyes de Britania (s. XII), en la fórmula céltica del traslado de Arturo a Ávalon para 
que este mismo se reponga de sus heridas. Aunque es en otra obra del mismo autor, Vita Merlini (1148), cuando se presenta a Morgana como una mujer sabia, la mayor de nueve hermanas, quien las educa no solo en la magia sino también en las ciencias, mientras que nada sugiere el parentesco entre Arturo y Morgana.

Morgana ha estudiado las virtudes medicinales de todas las plantas para aliviar los sufrimientos del cuerpo; la maestra en el famoso arte de la metamorfosis y con el que surca el aire con sus alas nuevas, como Dédalo. Curará al rey herido (Arturo) para permitirle volver entre los suyos y reconquistar Bretaña (HarfLancner, 2003: 802-89).

Además, con referencia a una de sus características más latentes, el término fay, hada, en el libro XIX, el capítulo "De incredulis", Burchard de Worms explica que existen dos tipos de mujeres sobrenaturales: las Parcas y seres misteriosos que solo se designan como hadas. Según la cultura culta, el hada se relaciona con la diosa del destino (=Parcas), mientras que en la cultura popular con la divinidad silvestre ligada al culto de la abundancia y de la fertilidad.

Las hadas habrían obtenido parte de sus rasgos de las Parcas de la mitología grecorromana. Tanto unas como otras poseen dotes adivinatorias y poderes sobrenaturales. La imagen de las tres Parcas hilando el destino de los hombres es frecuente en estos mitos. De esta misma manera se presenta también a Morgana: "Morgana bordaba sentada junto a la lumbre" (Miles, 1999: 254).

Pero aparece también como un hada que determina la vida del hombre, ya que su rasgo de fata, Morgana la Fay, lleva implícito este poder. “La definición más precisa sería que las hadas son una suma de las características de las Parcas, de las ninfas y de las diosas-madre de la religión gálica" (Harf-Lancner, 1984: 18).

Desde el siglo XIII con el auge de las obras del ciclo artúrico, cuyo estandarte es Chrétien de Troyes y sus novelas (Lancelot, el caballero de la carreta; Perceval, el cuento del Grial, Erec et Enide), Morgana se convertirá en la hermana del rey Arturo y la encargada de llevarlo a Ávalon. Chretien, influenciado aún por la cultura céltica, muestra al personaje como maga y sabia, unida también a la isla de Ávalon. En la isla dirige al grupo de hadas que está a su servicio y todas se muestran tal y como son. No admiten las órdenes de los hombres ni se someten a ellos. Son libres de pensar y hacer lo que quieran. No existe la noción del pecado cristiano ni del tiempo ni del espacio. En este ámbito restringido predominan los valores femeninos, mientras que fuera de él, el mundo pertenece al hombre y a sus deseos.

En su primer romance del ciclo artúrico, Erec et Enide (Chrétien, 1176), Morgana, el hada que reina con su amigo Guingamar en la isla de Ávalon, conserva sus poderes 
de sanación y prepara un ungüento para que Arturo se lo dé a Eric con el objetivo de curar sus heridas.

La tradición pagana prevalece en la obra Ginebra, el poder de la Gran Diosa, la libertad de la mujer presente en Camelot, en el País del Verano, incluso la de la hermana de Arturo. El personaje de Morgana sigue a la Madre -aquí se diferencia de Ginebra en cuanto a su cometido, para vengar lo que los hombres obligan a hacer a las mujeres: casarse con hombres mayores que no quieren o mandarlas a un convento para no tener que mantenerlas. Desde pequeña, su misión ha sido manejar a los hombres y destruirlos gracias a sus poderes mágicos. Lleva a cabo su venganza en la obra cuando hiere a Arturo, vestida como un caballero negro que se enfrenta a él en un torneo que este mismo organiza para celebrar la reconciliación entre Ginebra y él.

\begin{abstract}
Se convierte en la imagen inquietante del deseo femenino, la portavoz de las mujeres. De este modo, las mujeres, sumisas y relacionadas con la casa, una vez en el valle encantado que había creado Morgana, se convertirán en mujeres libres. Dentro del mismo, llevan una vida de placeres sensuales y se olvidan de los valores viriles. El hada Morgana encarna la amenaza de una mujer dominante y castradora y el peligro de los valores masculinos (Harf-Lancner, 2003: 85-89).
\end{abstract}

Con la expansión del cristianismo, la libertad de la mujer en este espacio mágico será condenada en las obras del siglo XIII, en la moral y en los valores caballerescos. En La búsqueda del Santo Grial (Anónimo, 1215-1230) ya no es una sabia y sanadora, sino que es una bruja, una arpía que intenta destruir los valores masculinos. Se convierte, pues, en el personaje, junto a Ginebra, antagónico del rey Arturo.

2.3. Morgana / Ginebra / Matriarcado

Si Morgana ha sido el estandarte de la destrucción de Camelot y de su hermanastro Arturo, su relación con Ginebra, también es otro de los puntos a resaltar. A pesar de que Ginebra haya cometido adulterio, su imagen ha sido menos degradada que la de Morgana. La reina se opone a ella como una diosa, una novia florida que representa la primavera, el despliegue de la vida y de las plantas, La boda de Sir Gauvain y la dama Ragnall, del poema Sir Gauvain y el caballero verde.

Normalmente, la relación entre ambas es destructiva. Morgana odia profundamente a Ginebra porque la separa de quien está enamorada y embarazada, Guingamor ${ }^{1}$. Hará todo lo posible por destruir tanto a su hermanastro como a ella.

En el caso que nos ocupa, no se odian porque no existe un pasado entre ellas que haga latir ese sentimiento; al contrario, en un principio Ginebra se apoya en Morgana para

1 Personaje relacionado siempre con el Otro Mundo. Por una parte, es el señor de la Isla de Avalón; por otra, las mujeres a las que ama, en los diferentes textos, suelen ser hadas. Es, por ejemplo, el amante de Morgana (Carlos Alvar, 1991: 216). 
confesar sus problemas con Arturo y no poder engendrar una hija, una heredera del País del Verano. Aunque provoca varias desgracias en la vida de la reina: la muerte de su hijo, el incesto con su hermanastro, el robo de la vaina de Excalibur, la degradación del rey, del matrimonio y del equilibrio de Camelot, Ginebra la defenderá como mujer, "ya que todas se deben proteger entre sí, y la librará de ser quemada en la hoguera" (Ginebra, 1999: 325-329).

Los caminos que siguen para defender la libertad de la mujer, simbolizado en la Gran Diosa son diferentes.

Morgana crea El Valle sin Retorno, Le Vals Sans Retour, que será un lugar donde los caballeros serán obligados a permanecer por infidelidad amorosa hasta que un caballero leal a su amor los libere (Lanzarote, restablece el orden moral masculino). Además, será su refugio particular ya que aquí se ocultará de la búsqueda que realizaron los caballeros de Arturo al ser secuestrado². Como se ha mencionado anteriormente, intenta vengarse de todos los hombres, más concretamente de su hermano con la intención de hacerle pagar el daño causado a su madre y, por tanto, a todas las mujeres. A lo largo de la novela, no podrá ser localizada debido a sus artes mágicas que determinan la desaparición del valle mediante niebla, bruma y lluvia.

Por su parte, Ginebra, representa tres símbolos tradicionalmente relacionados con Arturo:

a. El País del Verano, el poder de las mujeres, la tradición de la Diosa Madre frente a otros reinos donde imperan los poderes masculinos. Ávalon, el País del Verano, es descrito como Otro Mundo, un retiro apacible y saludable, sin noción del pecado original, donde los elementos arcaicos celtas permanecen vivos. No existe ni el tiempo ni el espacio, tampoco la muerte está presente.

b. La Tabla Redonda también sería gobernada por Ginebra. La mayor parte de las versiones, incluso, la más conocida, La Vulgata (Anónimo, s. XIII), había sido dirigida y creada por Arturo y sus doce caballeros. En la obra, la Diosa, la Gran Madre, regala a la primera reina del País del Verano, "la doncella más valiente y bella" (Ginebra, 1999: 127), los mejores guerreros para proteger al reino y a sus habitantes. Arturo necesita conquistarla para poder regirla y tener bajo su mando a todos los caballeros, lo cual implica derrocar el poder de las mujeres.

c. La espada Excalibur, que será entregada a Arturo como regalo de bodas junto con su vaina o funda enjoyada, el escudo y la lanza de la Reina. En las versiones patriarcales nada de esto pertenecía a una mujer.

2 Morgana se relaciona a menudo en los romances artúricos con la desaparición de un héroe en el otro mundo. En la variante del cuento melusiniano entrena a Guigamor en su reino y lo retiene ahí durante un tiempo (Harf-Lancner, 2003: 66-70). 
En diversas partes del libro, los comentarios de Ginebra, de la Madre (la voz de la autora) y de su asistenta Ina realizan referencias al matriarcado, a las tradiciones celtas, a los derechos de la mujer frente a los injurias por parte de aquellos (Merlín, los sacerdotes) que quieren acabar con esta tradición: “¡Yo no pertenezco a Arturo! masculló-. ¡En el País de la Madre, mi cuerpo me pertenece!”(Ginebra: 1999, 240).

Si en el siglo XII las mujeres mantenían una cierta individualidad, en el siguiente, el amor cortés será sustituido por el Grial, la búsqueda del caballero perfecto espiritualmente y el desprecio a todo acto realizado por la mujer: Morgana sabia, sanadora y curandera se convierte en una bruja malvada sin escrúpulos; Ginebra, una gran reina hasta la llegada de Lanzarote, es comparada con Eva, "una mujer pecadora que tienta a Adán a desobedecer a Dios lo que conlleva sufrir el pecado original" (Ginebra, 1999: 394); Putifar y Jezabel "la que denominan Señora, su sacerdotisa, la gran ramera que vive como Jezabel en su isla del lago" (Ginebra, 1999: 70). Todas las mujeres que aparecen en el libro son retratadas por los curas, sacerdotes y seguidores de la Iglesia católica como los personajes femeninos proscritos de la Biblia, unas pecadoras y seguidoras de Satán, que engañaron a sus respectivos maridos para conseguir lo que ellas anhelaban: el poder sobre cualquier hombre. "Cuando se proscribe la ley de la Madre y las mujeres se encuentran sometidas al yugo de los hombres" (Ginebra, 1999: 190), toda mujer era vista como pecado, mientras que los caballeros poseían un aspecto místico.

El derrocamiento de la Diosa a favor de un dios masculino fue el resultado de una tenaz conspiración de los varones -realeza, clases sacerdotal y clases altas (jefes militares, funcionarios,...) - que habían logrado el poder y el control social y deseaban mantenerlo en sus manos (Rodríguez, 2000: 321-343).

La tradición expone que es la reina quien elige a su rey; ella tiene la libertad de decidir sin depender de un hombre; ella es quien elige a su paladín, el cual llegará a ser su marido y su rey: "Las reinas y mujeres representan la presencia de las diosas que tiempo atrás fueron adoradas en estas islas" (Matthews, 1992:...).

"Vuestra madre ha gobernado este reino...de Aquella que es la Madre de todos nosotros" (Ginebra, 1999: 45). La matrilinealidad es común en muchos pueblos como en algunas tribus de Brasil, en Indonesia y en la antigua Irlanda, donde la figura femenina es mitificada en las leyendas y también se caracterizan por ser reinas guerreras.

Sobre todo, los pensamientos de la autora configuran su visión crítica de la expansión del cristianismo y el daño que ha estado haciendo a los pueblos, a su cultura y a sus tradiciones: "los cristianos se revolvieron contra la Gran Madre y derribaron los altares donde se adoraba" (Ginebra, 1999: 45); “Dios ha prohibido que las mujeres manden a los hombres. Los hombres fueron hechos a su imagen...las mujeres son obra del 
demonio y ofende a la voluntad de Dios" (Ginebra, 1999: 80); “los cristianos quieren derrocar a la Diosa e imponer la ley de los hombres” (Ginebra, 1999: 83).

La defensa del matriarcado presente mediante la explicación de la tradición celta, del periodo en el que las Diosas eran las predominantes, respetadas y adoradas por encima de los dioses y de los hombres: "El Cuerpo de la Madre...La sangre ${ }^{3}$ y la leche de la Madre...el amor de la Madre que se derrama sobre el mundo" (Ginebra, 1999: 137).

En la época del Paleolítico, la mujer se consideraba una diosa que se creía que era quien daba y quitaba la vida. Se conocía como la Diosa Madre y la Diosa Muerte. Toda la vida de cualquier ser vivo, de la naturaleza pasaban por sus manos; la mujer también poseía conocimientos de la vida y el desarrollo de las plantas, convirtiéndose en experta del cultivo y la recolección de frutos y plantas; con ayuda de estas últimas, adquirió métodos para curar enfermedades y heridas; cada una de ellas tenían claro su papel en la sociedad; se ayudaban entre ellas y no existía el ansia de poder. La sociedad matriarcal descansaba en un orden social que tanto el hombre como la mujer sabían perfectamente el papel que debían de realizar. Todo era armonía y equilibrio (Rodríguez, 2000: 203-235)

La mujer fue inventora del control del fuego, de los utensilios de piedra, de los procesos culinarios, la horticultura y la agricultura, la domesticación de algunos animales, prácticas curativas, rituales religiosos,...

2.4. Morgana / Arturo

El leitmotiv del viaje de Arturo y Morgana a la isla de Avalón aparece ya en el siglo XII en todas las obras de materia artúrica: el ciclo de la Vulgata que consta de cinco partes (Historia del Grial, Merlín, Lanzarote del Lago, La búsqueda del Santo Grial y La muerte del rey Arturo); las obras de Chrétien de Troyes (Erec y Enide; Lancelot, el Caballero de la Carreta; Perceval, el cuento del Grial), entre otras. Se exponía como la recuperación del rey para volver a reinar a los bretones y librarlos de los invasores sajones, siendo ella, la encargada de curar sus heridas y protegerlo. Dicho recurso será quebrado por la autora. Quien cura las heridas del rey será Ginebra como reina y gobernadora de Avalón, hasta ese momento lugar de culto y protectorado de Morgana.

Otra variante a tener en cuenta es la razón de la enfermedad y caída del rey Arturo. Normalmente ha estado vinculado con el amor adúltero entre Lanzarote y Ginebra y su posterior descubrimiento por parte de él mismo. Sin embargo, en la obra, el incesto con su hermana es lo que provoca tal destrucción. Como hemos mencionado anteriormente, el objetivo de su hermanastra era derrotar a los hombres cuyo estandarte era Arturo, ya sea por el hecho de ser hombre, ya sea por ser seguidor de una religión contraria a la

3 El color rojo debió simbolizar el color de los órganos regeneradores de la Madre; en el arte prehistórico el rojo simboliza la sangre y la vida; el triángulo pubiano remarcado en la escultura de la Diosa simbolizaba la regeneración (Rodríguez, 2000: 155-170). 
de la Madre. En el libro, se explica la "venganza" que lleva a cabo hacia la represión de los hombres. Su misión ha sido adquirir poderes mágicos para manejar a los hombres y destruirlos.

Una variante del cuento melusiniano se encuentra el de "morganiana", la desaparición de un héroe, su entrenamiento en su reino donde lo retiene para que esté bajo sus encantos y tenga poder sobre él. Si en dichos cuentos, versiones es un caballero quien es secuestrado por Morgana, aquí se trata de Arturo. Lo lleva a su reino durante un tiempo para anularlo como personaje masculino y le absorbe toda su voluntad, incluso manteniend relaciones y dando a luz a un hijo incestuoso.

\section{CONCLUSIÓN}

El personaje de Morgana es mucho más complejo que una simple "bruja malvada" que intenta derrocar el reino de Camelot y a su rey, Arturo. En sus primeras apariciones, simboliza una de las diosas célticas, la continuidad de la tradición de los antiguos, sabedora de los conocimientos de la Madre Tierra, de una época donde la mujer posee el respeto y el valor que nunca tendría que haber perdido. Una mujer poseedora de una gran sabiduría en diversas artes y ciencias: medicina (conocimiento de las plantas y su uso para la curación de las heridas y enfermedades), las matemáticas, la naturaleza, la magia,...Todo ello lo había ido trasmitiendo a otras generación de mujeres, si contamos con su labor de educar a sus ocho hermanas pequeñas, con las hadas y otras heridas por amor que vivían con ella en Avalón. No cabe olvidar uno de los papeles más importantes a los que estaba destinada: proteger a un gran rey, curarlo y darle el aliento suficiente para poder mantener la paz en su país.

El cambio demoledor que han ocasionado a Morgana, incluyo también a Ginebra, no es objetivo en ningún sentido. Ninguna sociedad, ninguna religión no puede derribar a un personaje ya sea real o ficticio por el simple hecho de ser mujer. Si durante la época del Paleolítico, la matrilinealidad era la opción imperante, quedando en la actualidad algunos pueblos con dicha cultura. Cada persona ya sea hombre o mujer sabía cuál era su papel en la sociedad sin que por ello existiese una pretensión por establecer su poder sobre los demás. La naturaleza asociada a los estados de la mujer -gestación, menstruación, ciclos de vida y muerte-, también sufrió su particular ocaso al considerarlas a ambas como seres inferiores, pasivos y posesiones del hombre.

Miles, expresa la inquietud y la disconformidad a este tipo de pensamiento dando un giro de $180^{\circ}$ a un mito que ha seguido la línea patriarcal de condenar a las mujeres como simples objetos y a los hombres como libres, activos y poseedores del poder y conocimiento. Todas las mujeres de la obra son autónomas, libres, fuertes, no representan el emblema de propiedad del hombre. Ginebra, es la reina del Camelot, la guardiana de Excalibur, de las tradiciones de la Madre Tierra; Morgana, elige qué 
camino ha de seguir en la vida; es el arquetipo de la liberación de la mujer. La obra permite romper con la idea que se hallaba anclada en el patriarcado y en la limitación de la mujer desde hace siglos.

\section{REFERENCIAS BIBLIOGRÁFICAS}

Agacinski, S., Política de sexos, Madrid, Editorial Taurus, 1999.

Alvar, C., El rey Arturo y su mundo. Diccionario de Mitología Artúrica. Madrid, Alianza Editorial, S. A., 1991.

Beteta Martín, Y., “Las heroínas regresan a Ítaca. La construcción de las identidades femeninas a través de la subversión de los mitos", Investigaciones Feministas (2009), vol 0, pp. 163-182.

Beteta Martín, Y., "Reescribir en femenino. La reelaboración del mito artúrico en Las Nieblas de Avalón de Marion Zimmer Bradley", EPOS, XXV (2009), pp. 197-214.

Campbell, J., Las máscaras de Dios: mitología primitiva, Madrid, Alianza Editorial, 1991.

Díez Celaya, R., La mujer en el mundo. Perspectiva antropológica. Visión histórica. La insurgencia feminista. El feminismo en el siglo XX. La tradición y las costumbres. El trabajo. La violencia. Madrid, Acento Ediciones, 1997.

Dunn Mascetti, M., Diosas, La canción de Eva, el Renacimiento del culto a lo femenino, Barcelona, Editorial Malsinet, 2008.

Gracia Noriega, J. Ignacio, El reino mágico de Arturo, Madrid, La Esfera de los Libros, S.L., 2009.

Fernández Guerrero, O., "El hilo de la vida. Diosas tejedoras en la mitología griega." Feminismo/s, Universidad Nacional de Educación a Distancia (La Rioja), (2012), pp. 107-125.

Gimbutas, M., Dioses y Diosas de la Vieja Europa, 7000-3500 a. C., Madrid, Editorial Siruela, 2014.

Harf-Lancner, L., Les Fées au Moyen Âge. Morgane et Mélusine. La naissance des fées, Paris, Editions Champion, 1984.

Harf-Lancner, L., Le monde des Fées dans l'occident médiéval, Paris, Hachette Littératures, 2003.

Matthews, J., Los misterios de la tradición artúrica, Madrid, Ediciones Temas de Hoy, 1992.

Miles, R., Ginebra, la Reina del País del Verano, New York, Crown Publishers, 1999. Posadas, C., Courgeron, S., A la sombra de Lilith, Barcelona, Editorial Planeta, 2004. Rodríguez, P., Dios nació mujer, Barcelona, Ediciones Barcelona, 1999. 
Van Gennep, A., La formación de las leyendas, Barcelona, Editorial Alta Fulla, 1982. Zarandona, J. Miguel, Cuaderno de Camelot. Cultura, literatura y traducción artúrica. Soria, Ediciones de la Excma. Diputación Provincial de Soria, 2002. 